\title{
Effectiveness of Temporal Augmentation Using a Calvarial Onlay Graft during Pterional Craniotomy
}

\author{
Yoon Soo Kim ${ }^{1}$, Hyung Suk Yi ${ }^{1}$, Han Kyu Kim², Yea Sik Han ${ }^{1}$ \\ ${ }^{1}$ Department of Plastic and Reconstructive Surgery, Kosin University College of Medicine, Busan; ${ }^{2}$ Department of Neurosurgery, Bundang \\ Jesaeng General Hospital, Seongnam, Korea
}

Temporal hollowing occurs to varying degrees after pterional craniotomy. The most common cause of temporal hollowing is a bony defect of the pterional and temporal regions due to the resection of the sphenoid ridge and temporal squama for adequate exposure without overhang. The augmentation of such bony defects is important in preventing craniofacial deformities and postoperative hollowness. Temporal cranioplasty has been performed using a range of materials, such as acrylics, porous polyethylene, bone cement, titanium, muscle flaps, and prosthetic dermis. These methods are limited by the risk of damage to adjacent tissue and infection, a prolonged preparation phase, the possibility of reabsorption, and cost inefficiency. We have developed a method of temporal augmentation using a calvarial onlay graft as a single-stage neurosurgical reconstructive operation in patients requiring craniotomy. In this report, we describe the surgical details and review our institutional outcomes. The patients were divided into pterional craniotomy and onlay graft groups. Clinical temporal hollowing was assessed using a visual analog scale (VAS). Temporal soft tissue thickness was measured on preoperative and postoperative computed tomography (CT) studies. Both the VAS and CT-based assessments were compared between the groups. Our review indicated that the use of an onlay graft was associated with a lower VAS score and left-right discrepancy in the temporal contour than were observed in patients undergoing pterional craniotomy without an onlay graft.

Keywords Craniotomy / Bone transplantation / Temporal bone

\author{
Correspondence: Yea Sik Han \\ Department of Plastic and \\ Reconstructive Surgery, Kosin \\ University College of Medicine, 262 \\ Gamcheon-ro, Seo-gu, Busan 49267, \\ Korea \\ Tel: +82-51-990-6131 \\ Fax: +82-51-990-3005 \\ E-mail: hanplastic1@naver.com
}

No potential conflict of interest relevant to this article was reported.

Received: 19 Aug 2015 • Revised: 29 Feb 2016• Accepted: 4 Mar 2016

pISSN: 2234-6163 • elSSN: 2234-6171 • http://dx.doi.org/10.5999/aps.2016.43.2.204 • Arch Plast Surg 2016;43:204-209

\section{INTRODUCTION}

Pterional craniotomy is the most commonly used neurosurgical approach in the management of cerebral aneurysms, frontal and temporal arterovenous malformations, traumatic cerebral hemorrhage, and brain tumor resections. A common cosmetic concern following pterional craniotomy is temporal hollowing, which can occur to varying degrees of severity [1]. A retrospective review was performed of 56 patients who underwent pterional craniotomy at the Kosin University Gospel Hospital between March 2009 and November 2013. Preoperative and postoperative tissue thickness was compared using axial computed tomography (CT) images. Temporal hollowing often occurs within six months of surgery and can be a significant cosmetic concern. It can be associated with localized pain in severe cases, due to the indentation of the temporalis muscle and adhesions to the dura [2]. It can also result in a delayed return to daily activities and adverse rehabilitation outcomes. With improvements in 
neurosurgical outcomes, postoperative cosmesis has received an increased emphasis in cranial operations. Several methods to prevent temporal hollowing following pterional craniotomy have been suggested, all with specific limitations $[3,4]$. In order to prevent temporal hollowing following pterional craniotomy, we have developed a method of temporal augmentation using a calvarial onlay graft as a single-stage neurosurgical reconstructive operation in patients requiring pterional craniotomy. In this report, we describe the surgical details and review our institutional outcomes.

\section{IDEA}

A retrospective review was performed to identify patients who underwent calvarial onlay grafting during pterional craniotomy at the Kosin University Gospel Hospital between March 2009 and November 2013. We selected patients who were followed up for at least six months and also had complete inpatient and outpatient medical records. Preoperative and postoperative photographs were used for the subjective assessment of temporal hollowing, which was evaluated on a visual analog scale (VAS) from 0 (no deformity) to 10 (severe temporal hollowing) at six months postoperatively. This study was carried out by three plastic surgeons and one neurosurgeon who were blinded to the operation. Preoperative and six-month postoperative cranial CT scans were used to measure the soft tissue thickness over the temporal area. This thickness was defined as the shortest distance between the skin surface and a line drawn from the lateral orbital rim tangentially to the temporal bone. These measurements were made for both the temporal soft tissue that was operated on and the temporal soft tissue that was not operated on. A comparison was made between the operated and non-operated sides in each patient (Fig. 1).

Each operation began with both the neurosurgical and plastic surgery teams present in the operating room. Upon surgical preparation, the scalp incision was designed and executed by the plastic surgeons. The dissection was extended downwards until the superficial temporal fascia was encountered. This superficial fascia was then incised and reflected anteriorly, along with the frontal periosteum. This maneuver exposed the deep temporal fascia and the temporalis muscle, which were divided with a $1.5-\mathrm{cm}$ myofascial cuff retained along the superior temporal line.

With the soft tissue layers dissected and the lateral portion of the cranium exposed, the neurosurgeons created the craniotomy access and commenced the intracranial portion of the operation (i.e., tumor removal or hematoma removal). The cranial bone flap was held in saline-soaked gauze/solution until needed. For pterional craniotomy patients, the craniotomy flap was

\section{Fig. 1. Computed tomography (CT) images}

Temporal thickness was measured in postoperative CT images. Soft tissue thickness was measured perpendicularly (blue line) from the reference line extending from the lateral orbital rim to the tangential point on the temporal bone (white dotted line). Preoperative images are on the left, and six-month postoperative images are on the right. The axial images are from the end of the sphenoparietal suture level. $(A, B)$ This patient underwent pterional craniotomy on the left side, with onlay graft reconstruction (yellow arrow). Postoperative temporal hollowness was not observed to a significant extent in the onlay graft group when a comparison was made between the non-operated ( $u$ ) and operated sides (a).
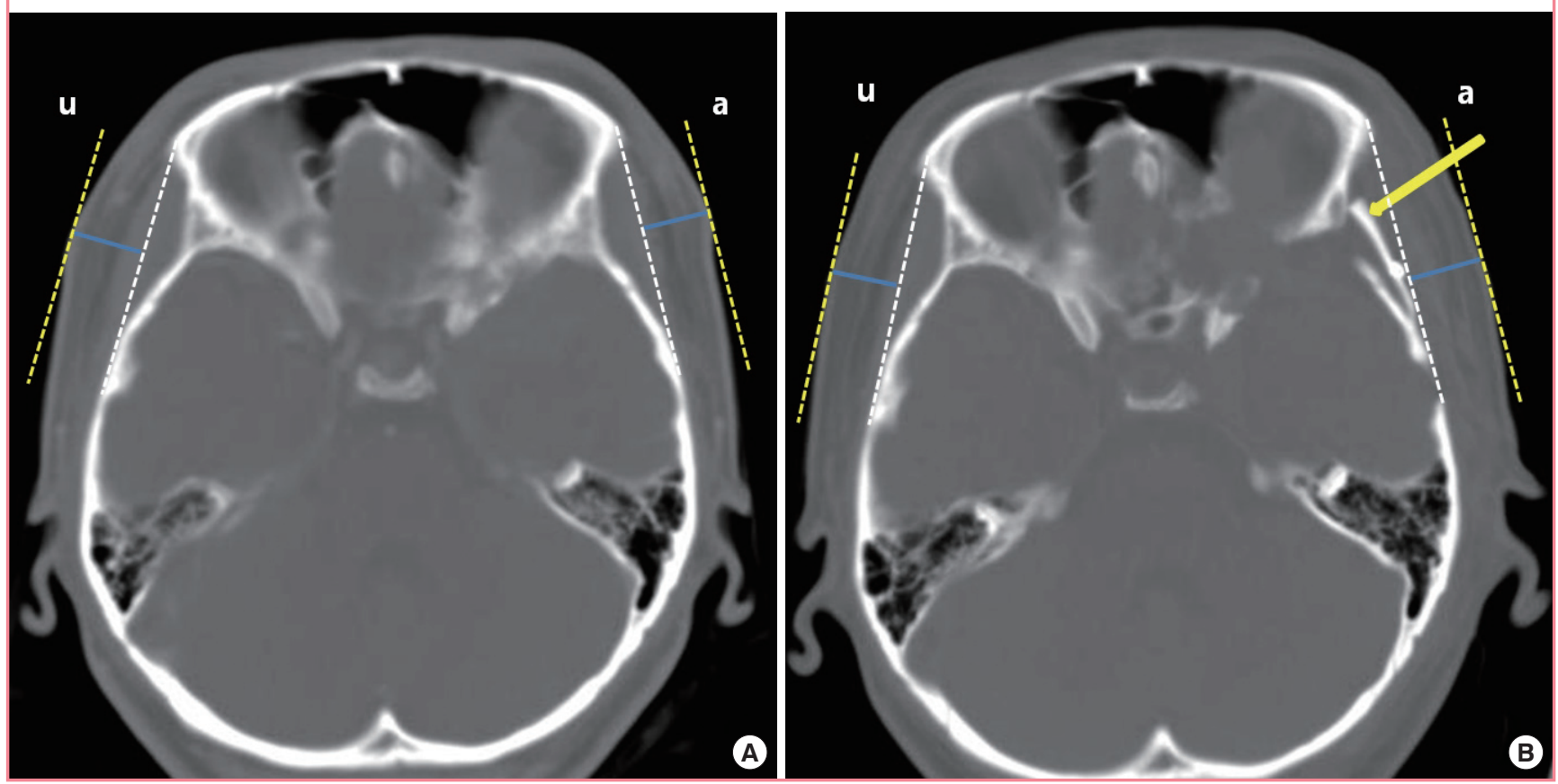


\section{Fig. 2. Surgical detail}

(A) A pterional and temporal skull defect is demonstrated with a red dotted line corresponding to where the bone was removed during burr hole placement and pretemporal sphenoid wing drilling. The cranial flap was divided into two parts. The upper bone flap was bisected, and the outer table of this flap (blue dotted line) was used to close the rest of the craniotomy defect. The inner table of the upper bone flap was used as source material for the onlay graft. (B) A calvarial onlay graft was secured to the bone flap with titanium screws. (C) The onlay graft was made to overlap the cranial bone flap in order to ensure an appropriate thickness. Onlay grafts were prepared to overcorrect for soft tissue atrophy following the operation. (D) The temporalis muscle was reapproximated to the myofascial cuff along the superior temporal line.
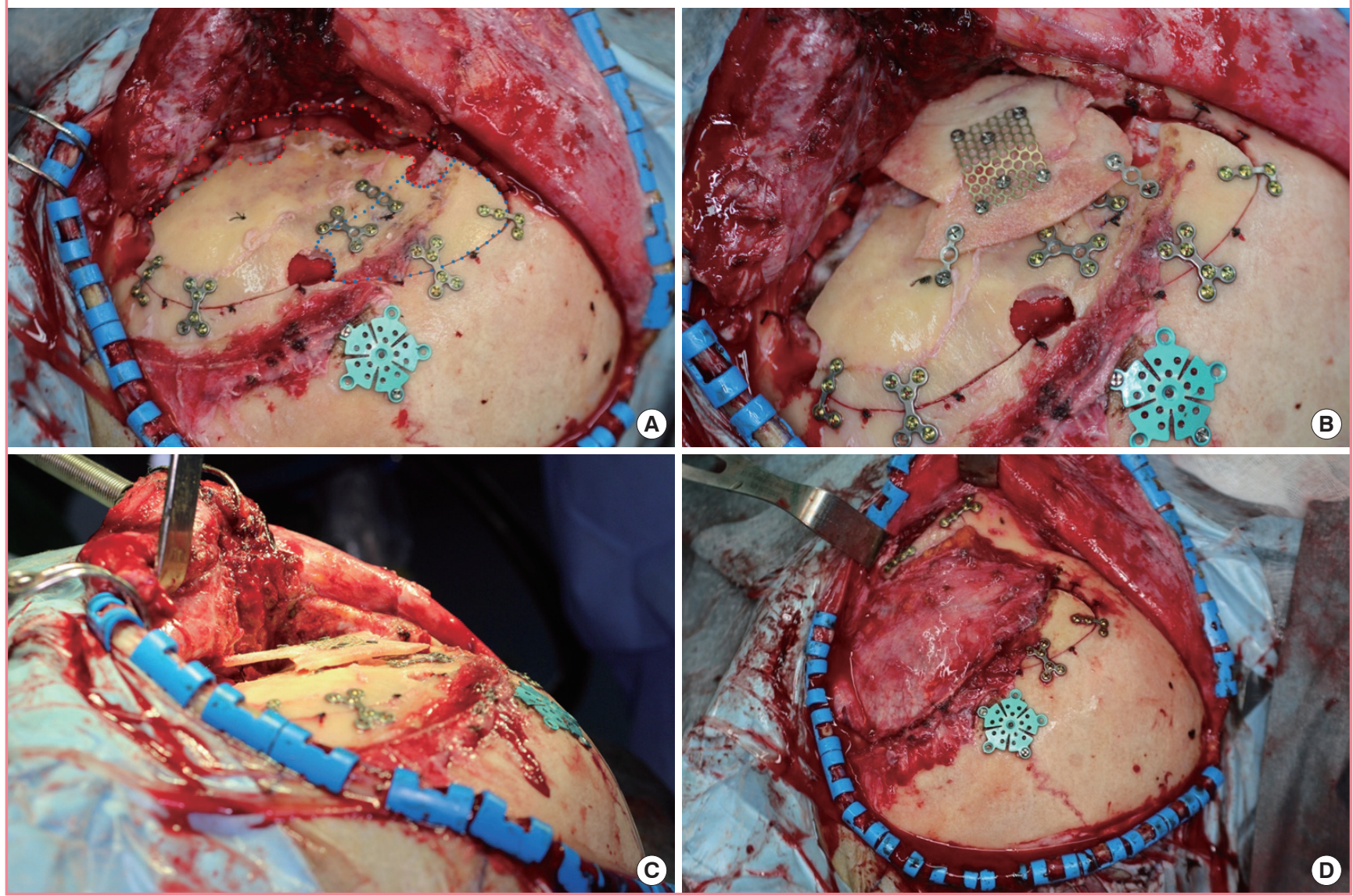

restored at the conclusion of the neurosurgical operation. For onlay graft patients, however, the cranial flap was prepared while the intracranial operation was still ongoing. The flap was divided into the upper third and the lower two thirds. The lower bone flap was fixed into the original position with plates and screws. The upper bone flap was bisected, and the outer table of this flap was used to close the rest of the craniotomy defect. The inner table of the upper bone flap was used as source material for the onlay graft.

The inner table was set aside, and the cranial defect was outlined on a sterile paper as a template. This template was brought over to the inner table, and the onlay graft was designed to be slightly larger than this defect to allow adequate coverage of the pterional and temporal defects. In addition, the onlay graft was made to overlap the cranial bone flap to offset atrophy in the postoperative temporalis muscle. Once the cranium layer was closed, the temporalis muscle was reattached to the myofascial cuff at the superior temporal line, using 3-0 Vicryl sutures. The scalp was closed primarily in layers (Fig. 2).

The mean follow-up period was 20 months. The mean onlay graft dimensions were $43.1 \times 34.8 \times 5.7 \mathrm{~mm}$.

The mean VAS score was $1.4 \pm 0.9$ for the onlay graft group, while the mean score was $5.8 \pm 2.2$ for the pterional craniotomy group. The mean score was significantly lower for the onlay graft group $(\mathrm{P}<0.05)$ (Fig. 3). On CT imaging, preoperative soft-tissue thickness was not significantly different between the operated and the non-operated sides $(\mathrm{P}>0.05)$. Postoperative temporal hollowness was not observed to a significant extent in the onlay graft group $(8.4 \pm 2.9 \mathrm{~mm}$ vs. $8.2 \pm 2.2 \mathrm{~mm}$ for the operated and non-operated sides, respectively; $\mathrm{P}=0.837$ ) (Table 1 ). As mentioned above, however, the pterional craniotomy group showed a statistically significant difference between the operated side and the non-operated side $(\mathrm{P}=0.006)$ (Table 2). 


\section{Fig. 3. Postoperative photographs at six months}

(A) This patient underwent pterional craniotomy without an onlay graft. The visual analog scale (VAS) score was 7. (B) This patient underwent only graft reconstruction. The VAS score was 1 . An asterisk $\left(^{*}\right)$ designates the operated side.
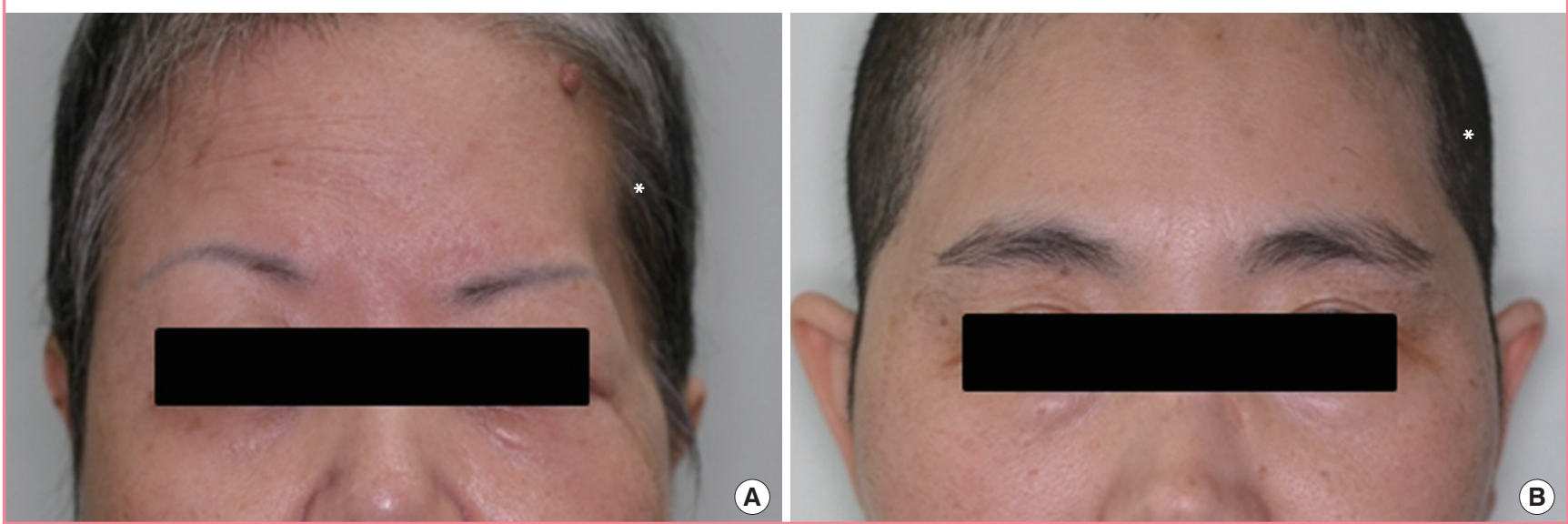

Table 1. Thickness of the temporal soft tissue in the onlay graft group

\begin{tabular}{|c|c|c|c|c|c|}
\hline \multirow{3}{*}{ Category } & \multicolumn{5}{|c|}{ Onlay graft group $(n=10)$} \\
\hline & \multicolumn{2}{|c|}{ Operated side $(\mathrm{mm})$} & \multicolumn{2}{|c|}{ Non-operated side (mm) } & \multirow{2}{*}{ P-value ${ }^{\text {a) }}$} \\
\hline & Median & IOR & Median & IQR & \\
\hline Preoperative & 8.63 & $5.19-11.85$ & 7.22 & $6.53-10.30$ & 0.949 \\
\hline Six months postoperatively & 8.20 & $7.02-10.71$ & 7.81 & $6.48-9.26$ & 0.837 \\
\hline
\end{tabular}

Table 2. Thickness of the temporal soft tissue in the pterional craniotomy group

\begin{tabular}{|c|c|c|c|c|c|}
\hline \multirow{3}{*}{ Category } & \multicolumn{5}{|c|}{ Pterional craniotomy group $(n=56)$} \\
\hline & \multicolumn{2}{|c|}{ Operated side (mm) } & \multicolumn{2}{|c|}{ Non-operated side $(\mathrm{mm})$} & \multirow{2}{*}{ P-value } \\
\hline & Median & IQR & Median & IQR & \\
\hline Preoperative & 8.95 & $6.80-10.71$ & 9.15 & $7.57-11.58$ & 0.519 \\
\hline Six months postoperatively & 4.36 & $1.11-6.45$ & 7.54 & $6.50-10.54$ & $0.006^{a)}$ \\
\hline
\end{tabular}

\section{DISCUSSION}

Temporal hollowing occurs to varying extents after pterional craniotomy. The most common cause of temporal hollowing is a bony defect of the pterional and temporal regions due to the resection of the sphenoid ridge and temporal squama for adequate exposure without overhang. The augmentation of such bony defects is important in preventing craniofacial deformities and postoperative hollowness. The primary goals of temporal augmentation are to provide appropriate coverage over the underlying intracranial soft tissue. In addition, the secondary goal of aesthetic restoration is to alleviate psychosocial impairments caused by temporal hollowing. Temporal cranioplasty has been performed using materials such as acrylics, porous polyethylene, bone cement, titanium, muscle flaps, and prosthetic dermis $[3,4]$. These methods are limited by the risk of damage to the adjacent tissue and infection, a prolonged preparation phase, the possibility of reabsorption, and cost inefficiency.

Calvarial onlay grafts use autologous tissue and provide optimal and rapid recovery after cranial defect reconstruction. This method has not been previously described in other papers. The graft is easy to prepare and sturdy, with a low risk of infection. At our institution, temporal augmentation is performed as part of a single-stage neurosurgical operation, thereby decreasing the need for a separate reconstructive operation with the additional costs and risks involved. 
Aside from bony loss, atrophy of the superficial temporal fat pad and temporalis muscle can be another cause of post-craniotomy temporal hollowing. The temporalis muscle may undergo atrophy after a number of physiological insults, including direct damage to muscle fibers, vessel damage, prolonged traction, inappropriate tension in the reattached muscle, and atrophy secondary to nerve damage [5-8]. Several studies have identified potential methods of reducing this postoperative atrophy. These approaches include preservation of the superficial temporal artery, ensuring that facial incisions do not damage the facial nerves, subfascial dissection to protect the frontotemporal branch of the facial nerve, reduced temporalis muscle traction during zygomatic bone dissection, retrograde subperiosteal dissection from the origin of the temporalis muscle to minimize the risk of damage to the deep temporal nerves and blood vessels, reattachment of the temporalis muscle using screws and sutures, and the elimination of periosteal dissection by elevating the muscle and bone as a single unit [2,5-7,9-11]. Complications of temporal augmentation using calvarial onlay grafts include donor-site morbidity from thinning of the lower cranial bone flap that is bisected and secured onto the original position, and over-correction from overlapping with the onlay graft. However, none of the patients in this study developed these complications during the follow-up period.

In this study, temporal hollowing was assessed using clinical photographs. Each photograph was evaluated by neurosurgeons and plastic surgeons using a VAS. Patients who underwent calvarial onlay grafts scored significantly lower $(1.4 \pm 0.9)$ than those who underwent pterional craniotomy $(5.8 \pm 2.2)$. The VAS scores indicated that calvarial onlay grafts were associated with a decreased amount of temporal atrophy and satisfactory postoperative symmetry in comparison with pterional craniotomy (Fig. 3). However, VAS scores may not be comparable across studies, and are only appropriate for distinguishing the degree of temporal hollowing within a single study involving a fixed group of observers.

On a physical exam, temporal hollowing after pterional craniotomy is easily appreciated, but cannot be quantified in a reliable manner because no obvious point of reference exists over the temporal contour. In the wider craniofacial literature, CT studies have been used for a more objective and quantifiable assessment of temporal hollowing. In order to evaluate temporal hollowing in the context of coronal incisions for non-intracranial pathology (i.e., facial bone fractures), Matic and Kim [12] used preoperative and postoperative $\mathrm{CT}$ studies to determine the effect that various levels of temporal muscle dissection had on temporal fat pad volume. In craniosynostosis patients, CT images have been used for the cephalometric evaluation of bony temporal hollowing, with the sella turcica as the reference point [13]. In this study, we chose to evaluate the partial thickness of the temporal soft tissue using the reference line described above (from the lateral orbital rim, extending tangentially to the temporal bone) as a proxy measure of temporal depression.

The CT-based measurements were commensurate with the degree of clinical temporal hollowness as reflected by the VAS scores. For instance, no statistically significant difference was found in temporal hollowing among patients who had undergone onlay grafts ( $8.20 \mathrm{~mm}$ [interquartile range, $7.02-10.71$ $\mathrm{mm}$ ] vs. $7.81 \mathrm{~mm}$ [interquartile range, $6.48-9.26 \mathrm{~mm}$ ] for the operated vs. non-operated sides) with a low mean temporal hollowness score $(1.4 \pm 0.9)$. Our review indicates that onlay grafts were associated with lower VAS scores and left-right discrepancies in the temporal contour than were observed in patients who underwent pterional craniotomy without an onlay graft.

During this operation, it is important that a sufficient myofascial cuff is left for reattachment of the temporalis muscle when the deep temporal fascia and the temporalis muscle are exposed during pterional craniotomy. The method of temporal augmentation using a calvarial onlay graft described in this study involves a large bony defect that must be covered, and it is difficult to completely prevent temporal hollowing due to atrophy of the temporalis muscle.

This study has several limitations. Due to the retrospective nature of the study design, we cannot eliminate the possibility of selection bias. Despite the small sample size, however, we were still able to find a statistically significant difference between the two groups. The study only assessed temporal hollowing in the context of craniotomy patients, and our results should not be generalized to temporal hollowing in different contexts. In addition, further research is needed to determine whether onlay grafts are effective in preventing temporal hollowing in patients undergoing repeated or bilateral craniotomy. Lastly, it is necessary to compare the degree of temporal hollowing in patients who underwent other methods of temporal augmentation. Nevertheless, this study is the first of its kind to report the outcomes of single-stage calvarial onlay grafts during neurosurgical operations for temporal augmentation. Through the clinical and radiographic evaluation of temporal hollowing, we were able to demonstrate that the use of onlay grafts was associated with temporal symmetry.

\section{REFERENCES}

1. Choudhry OJ, Christiano LD, Arnaout O, et al. Reconstruction of pterional defects after frontotemporal and orbitozygomatic craniotomy using Medpor Titan implant: cosmetic 
results in 98 patients. Clin Neurol Neurosurg 2013;115:171620.

2. Matsumoto K, Akagi K, Abekura M, et al. Cosmetic and functional reconstruction achieved using a split myofascial bone flap for pterional craniotomy: technical note. J Neurosurg 2001;94:667-70.

3. Rapidis AD, Day TA. The use of temporal polyethylene implant after temporalis myofascial flap transposition: clinical and radiographic results from its use in 21 patients. J Oral Maxillofac Surg 2006;64:12-22.

4. Badie B. Cosmetic reconstruction of temporal defect following pterional [corrected] craniotomy. Surg Neurol 1996;45: 383-4.

5. Kadri PA, Al-Mefty O. The anatomical basis for surgical preservation of temporal muscle. J Neurosurg 2004; 100:517-22.

6. Oikawa S, Mizuno M, Muraoka S, et al. Retrograde dissection of the temporalis muscle preventing muscle atrophy for pterional craniotomy: technical note. J Neurosurg 1996;84: 297-9.

7. Spetzler RF, Lee KS. Reconstruction of the temporalis muscle for the pterional craniotomy: technical note. J Neurosurg
1990;73:636-7.

8. Appell HJ, Gloser S, Duarte JA, et al. Skeletal muscle damage during tourniquet-induced ischaemia. The initial step towards atrophy after orthopaedic surgery? Eur J Appl Physiol Occup Physiol 1993;67:342-7.

9. Miyazawa T. Less invasive reconstruction of the temporalis muscle for pterional craniotomy: modified procedures. Surg Neurol 1998;50:347-51.

10. Ammirati M, Spallone A, Ma J, et al. An anatomicosurgical study of the temporal branch of the facial nerve. Neurosurgery 1993;33:1038-43.

11. Brunori A, DiBenedetto A, Chiappetta F. Transosseous reconstruction of temporalis muscle for pterional craniotomy: technical note. Minim Invasive Neurosurg 1997;40:22-3.

12. Matic DB, Kim S. Temporal hollowing following coronal incision: a prospective, randomized, controlled trial. Plast Reconstr Surg 2008;121:379e-385e.

13. Steinbacher DM, Wink J, Bartlett SP. Temporal hollowing following surgical correction of unicoronal synostosis. Plast Reconstr Surg 2011;128:231-40. 